\title{
() Institution animal care and use committees need greater ethical diversity
}

\author{
Lawrence Arthur Hansen
}

\section{Correspondence to Dr Lawrence Arthur Hansen, Department of Neurosciences and Pathology, University of California San Diego, 9500 Gilman Dr, La Jolla, CA, 92093-0624, USA; lahansen@ucsd.edu}

Received 27 July 2012 Revised 25 September 2012 Accepted 18 October 2012 Published Online First 6 November 2012

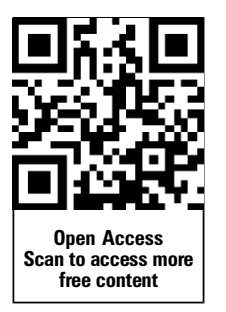

\section{ABSTRACT}

In response to public outrage stemming from exposés of animal abuse in research laboratories, the US Congress in 1985 mandated Institutional Animal Care and Use Committees (IACUCS) to oversee animal use at institutions receiving federal grants. IACUCs were enjoined to respect public concern about the treatment of animals in research, but they were not specifically instructed whether or not to perform ethical cost-benefit analyses of animal research protocols that IACUCs have chosen, with approval contingent upon a balancing of animal pain and suffering against a reasonable expectation of resultant human benefit. IACUCs have chosen not to make such ethical judgments but, rather, restrict themselves to an advisory role, often tweaking the details of animal-use protocols, but eventually approving all of them. This disinclination by IACUCs to take a broader ethical view of their authority and responsibilities may reflect a membership composition highly skewed towards animal researchers themselves $(67 \%)$ and institutional veterinarians $(15 \%)$, both with vested interests in continuing animal research. The resultant ethical monoculture may impair IACUC's ability to meet public concern for laboratory animal welfare. Psychological research has established that unconscious bias affects us all, that deliberations among the likeminded lead to adapting extremist positions, and that groupthink blinds organisations to alternatives that might be obvious to outsiders. Taken together, skewed IACUC membership composition and psychological research insights into unconscious bias and groupthink suggest that an infusion of ethical diversity by increasing the percentage of institutionally unaffiliated members on IACUCs would broaden their ethical perspectives and enable them to better address public concerns about laboratory animal welfare.

\section{INTRODUCTION}

Animal use in research and teaching is an ethically contentious issue. Institutional Animal Care and Use Committees (IACUCs) were mandated by Congress in 1985 in response to public concern about the welfare of laboratory animals stemming from exposés of abuse in federally funded research laboratories. ${ }^{1}$ Lawmakers stipulated that IACUCs should 'respect society's concerns regarding the welfare of animal subjects', but the extent to which they were intended to function as animal research ethics committees is unclear in the somewhat ambiguous wording of the legislation itself, and ensuing implementation regulations issued by the Public Health Service and the US Department of Agriculture. Animal protectionists hoped IACUCs would make ethical judgments and reject research protocols when their cost in animal suffering was high and the potential medical benefit to humans was low or non-existent. Some support for that hope can be found in IACUC protocol review requirements which instruct IACUCs to consider US government principles when reviewing protocols, one of which states 'procedures involving animals should be designed and performed with due consideration of their relevance to human or animal health, the advancement of knowledge, or the good of society'. ${ }^{2}$ IACUCs did not assume the role of ethics committees, however, and one national authority on how they function frankly acknowledges that relevance of proposed animal research to human disease is immaterial in their deliberations, and any research procedures, even if painful, can be approved. ${ }^{3}$ Rather than making ethical judgments, IACUCs have restricted themselves to technical or advisory roles focused on reworking the details of some animal-use protocols, but ultimately approving almost all of them.

While uncertainty persists about whether Congress intended IACUCs to function as animal ethics committees, one raison d'être for their creation was explicitly to 'meet the public concern for laboratory animal care and treatment important in assuring that research will continue to progress'. Longitudinal public opinion polling, however, shows increasing erosion of support for animal testing since the IACUC system was initiated. Overall opposition rose from 33\% to $43 \%$ between 2001 and 2011, while opposition among women (52\%) and the young (59\%) has become the majority view. Polls also show less public support for animal testing when it is not directly related to human medical benefit. ${ }^{5}$

The thesis of this article is that IACUCs could better serve the public interest in animal welfare and ensure continued popular support for animal research if their memberships were less skewed towards animal researchers and institutional employees. Better balanced IACUCs would lessen the risks of bias and groupthink and broaden their deliberations to include ethical dimensions of animal research.

\section{IACUC MEMBERSHIP COMPOSITIONS AND PROTOCOL APPROVAL RATES}

IACUCs at US research institutions are dominated by animal researchers and institutional employees whose livelihoods are linked to animal research. A recent study found that at 21 of the top 25 National Institutes of Health (NIH)-funded research institutions (data from four was insufficient for analysis), an average of $67 \%$ of IACUC members were animal researchers, and $15 \%$ were institutional veterinarians, many of whom also 
engaged in animal research. Ninety-three percent of IACUC chairpersons were animal researchers. ${ }^{6}$ It is not unduly cynical for animal welfare advocates to wonder if an animal-use committee system in which $82 \%$ of members and $93 \%$ of chairpersons have vested interests in continuing animal research might be subject to an approval bias.

The most comprehensive analysis of IACUC reviews found 98\% approval rates for in-house research protocols, but that when the same protocols were evaluated by blinded IACUCs from other institutions, $61 \%$ were judged as 'not very understandable' or 'not understandable at all', as having poor research designs and procedures, or as justifying the type and number of animals in a way that was 'not very convincing' or 'not convincing at all'. The authors concluded that 'IACUCs will rarely disapprove of protocols that other committees feel should be rejected'. ${ }^{7}$ Evidence that IACUCs often approve protocols which do not meet federal standards can also be found in Federal audits, government surveys of US Department of Agriculture laboratory inspectors, and USDA reports. ${ }^{6}$

The predominance of animal researchers, and the institutional veterinarians who support them on IACUCs, may also explain why IACUCs evolved into exclusively technical or advisory committees rather than ethics committees. The great majority of IACUC members have, through their career choices, made clear their allegiance to animal experimentation, and for them the ethical conclusion that the ends justify the means is self-evident. The resultant ethical monoculture of the IACUC committee mind may, therefore, be disinclined to revisit the issue in protocol after protocol. Recall that neither the original 1966 Animal Welfare Act, nor its 1985 amending to mandate the creation of IACUCs, were instigated by animal scientists, but rather, by public outrage over perceived mistreatment of laboratory animals.

\section{PERVASIVENESS OF BIAS IN HUMAN PSYCHOLOGY AND GROUPTHINK}

Human beings generally, and perhaps scientists especially, are inclined to flatter themselves that, compared with most people, we are free from judgment-distorting biases. Ironically, psychological studies have shown that we are biased towards seeing ourselves as less biased than average. ${ }^{8}$ Psychology research has contrarily demonstrated that bias is pervasive in us all, and that decision-influencing thoughts and feelings exist outside of our conscious awareness or control. ${ }^{9}$ We are also about twice as likely to seek information that supports our current view as we are to consider an opposing idea. ${ }^{10}$ And because changing our minds is painful, even when facts are presented which might lead us to do so, we scrutinise them more sceptically, and require more evidence from them than we do for facts that allow us to believe our favoured conclusions. ${ }^{11} 12$

One strategy for ameliorating judgment bias is to bring multiple perspectives to bear on a decision. This method of error reduction only works, however, when the judgments are independent. If observers share a bias, the aggregation of their judgments will not only fail to reduce it, but will actually make it worse. ${ }^{13}$ When groups of like-minded people get together, they make each others' views more extreme rather than less. ${ }^{14}$

In arriving at consensus in like-minded groups, the opinions sought and shared are those that reinforce, rather than challenge, the favoured conclusion. ${ }^{15}$ Group discussions among people who start out with similar views render the participants blind to alternatives which would seem obvious to outsiders. ${ }^{9}$ Neuroscientists employing sophisticated neuroimaging techniques have found that the areas of the brain responsible for perception are altered by social influences, that is, what we perceive depends on what others see, and that the cost of registering a dissenting opinion in a homogeneous group is emotional distress, as evidenced by activation of the amygdala, the avoidance of which further encourages groupthink. ${ }^{16}$

Experts in the study of bias are pessimistic about our ability to overcome them, and recommend that once we have acknowledged the biases we bring to any group, we have to adjust for them, and a balance of biases may be the best we can achieve. ${ }^{9}$ Encouragingly, though, researchers have found that mere awareness of a dissenting voice in a group is enough to alter cognitive processes and yield better judgments. ${ }^{17}$ Dissenting opinions in IACUC deliberations are unlikely to be heard, however, when there is too great an imbalance between researchers and institutional representatives on the one hand, and unaffiliated members on the other. One interview-based Canadian study about the effectiveness of animal research ethics committees reported that three of the six community representatives on the committees found the atmosphere intimidating, two of the six had negative experiences with other committee members, and common problems were the difficulties of being an 'outsider' and a perceived lack of appreciation for their contribution. ${ }^{18}$ Participants in another interview-based study of US IACUCs expressed a wish for a broader range of people on the committee, such as an ethicist, or more community members. ${ }^{19}$

Interestingly though, and perhaps counterintuitively, a similar interview-based study of animal ethics committees in Sweden, where their composition is almost equally balanced between scientists and laypersons, reported that most interviewees agreed that the committees were discussing the 'wrong' question. They all knew that they were supposed to be conducting cost-benefit evaluations, but instead, just like US IACUCs, they addressed almost exclusively experimental methodology. They found themselves discussing refinement questions in great detail, but not research purposes, replacement and reduction. ${ }^{20}$ These findings seem to imply that even with a better balance between laypersons and scientists with vested interests in propagating animal research, institutional-based animal research ethics committees will not address broad ethical issues. The authors of the Swedish study attribute this reluctance to 'situated ethics' in which context, environment and unspoken strategies are created to make ethical dilemmas easier to handle by avoiding altogether fundamental questions, such as whether it is ever 'right' or 'wrong' to wilfully inflict pain and suffering upon a sentient being. Even if scientific experts are not a majority, they have power over the agenda, and the priority of interpretation belongs exclusively to the scientific ideals, to the exclusion of deeper ethical questions about research purposes and animal suffering. ${ }^{20}$

\section{CONCLUSIONS}

The NIH acknowledges that 'the validity of IACUC actions is always predicated on the existence of a properly constituted IACUC' ${ }^{21}$ While this statement literally refers to research facilities' ability to conform to minimum IACUC membership requirements, in a larger sense it encourages us to ask what a 'properly constituted IACUC' would look like, and what would its valid actions be. IACUCs have never been explicitly directed to make ethical cost-benefit determinations in deciding to approve or disapprove animal-use protocols, but neither have they been discouraged from doing so by legislation or regulation. IACUCs have chosen not to function as animal ethics committees because of their overwhelming proportions of animal researchers, laboratory animal veterinarians and other members with vested interests in seeing animal experiments approved. But since IACUCs are the only mechanism available for 
addressing the ethics of animal research, they should broaden their purely technical scope to consider larger ethical issues, including harm-benefit determinations. Infusing greater ethical diversity into their memberships would further this goal.

Funding None.

Competing interests None.

Provenance and peer review Not commissioned; externally peer reviewed.

Open Access This is an Open Access article distributed in accordance with the Creative Commons Attribution Non Commercial (CC BY-NC 3.0) license, which permits others to distribute, remix, adapt, build upon this work non-commercially, and license their derivative works on different terms, provided the original work is properly cited and the use is non-commercial. See: http://creativecommons.org/ licenses/by-nc/3.0/

\section{REFERENCES}

1 Holden C. A pivotal year for lab animal welfare. Science 1986;232:147-50.

2 Health Research Extension Act of 1985. Public law 99-158. Bethesda, MD, USA National Institutes of Health, 1985. http://grants.nih.gov/grants/olaw/references/ hrea1985.htm

3 Carbone L. What animals want: expertise and advocacy in laboratory animal welfare policy. New York: Oxford University Press, 2004:182-6.

4 Food Security Act of 1985. Public Law 99-198. 102,7 USC 2131. USA, 1985.

5 Goodman JR, Casey BA, Cherry E. Mounting opposition to vivisection. Contexts 2012;11:68.

6 Hansen LA, Goodman JR, Chanda A. Analysis of animal research ethics committee membership at american institutions. Animals 2012;2:68-75.

7 Plous $\mathrm{S}$, Herzog $\mathrm{H}$. Reliability of protocol reviews for animal research. Science 2001;293;608-9.
8 Pronin E, Gilovich T, Ross L. Objectivity in the eye of the beholder: divergent perceptions of bias in self versus others. Psychol Rev 2004; ;11:781-99.

9 Heffernan M. Willful blindness: why we ignore the obvious at our peril. Walker Company 2011:11-12

10 Hart W, Albarracín D, Eagly A, et al. Feeling validated versus being correct: a meta-analysis of selective exposure to information. Psychol Bull 2009;135:555.

11 Pyszcynski T, Greenberg J, Holt K. Maintaining consistency between self-serving beliefs and available data: a bias in information evaluation. Pers Soc Psychol Bull 1985;11:179-90.

12 Ditto PH, Lopez DF. Motivated skepticism: use of differential decision criteria for preferred and nonpreferred conclusions. J Pers Soc Psychol 1992;63:568-84.

13 Kaheman D. Thinking, fast and slow. New York: Farrar, Straus and Giroux, 2011:84.

14 Sunstein CR. Going to extremes: how like minds unite and divide. Oxford: Oxford University Press, 2009:1-6.

15 Tesser A, Rosen S. Similarity of objective fate as a determinant of the reluctance to transmit unpleasant information: the MUM effect. J Pers Soc Psychol 1972;23:46-53.

16 Berns GS, Chappelow J, Zink CF, et al. Neurobiological correlates of social conformity and independence during mental rotation. Biol Psychiatry 2005:58:245-53.

17 Nemeth C, Mayseless 0, Sherman J, et al. Exposure to dissent and recall of information. J Pers Soc Psychol 1990;58:429-37.

18 Schuppli CA, Fraser D. Factors influencing the effectiveness of research ethics committees. J Med Ethics 2007;33:294-301.

19 Graham K. A study of three IACUCs and their views of scientific merit and alternatives. J Appl Anim Welf Sci 2002;5:75-81.

20 Ideland M. Different views on ethics: how animal ethics is situated in a committee culture. J Med Ethics 2009;35:258-61.

21 Maintenance of Properly Constituted IACUCs. Report No.: 97-03. Bethesda, MD, USA: National Institutes of Health, 1997. http://grants.nih.gov/grants/olaw/ references/dc97-3.htm 